\title{
Personalized Human Robot Interaction in the Unique Context of Rehabilitation
}

\author{
Ronit Feingold Polak \\ Ben-Gurion University of the Negev \\ polakr@post.bgu.ac.il
}

\begin{abstract}
Socially assistive robots (SARs) can help meet the growing need for rehabilitation assistance; We argue that personalization of humanrobot interactions in the context of rehabilitation is multi-layered, and needs to be frequently updated, as opposed to a single setting that might suffice in other contexts. In rehabilitation, personalization is not only important in order to establish engagement, but it is an essential component for the recovery of motor and cognitive abilities over a long-term interaction, and is an essential part of establishing trust between the patient and the SAR.
\end{abstract}

\section{CCS CONCEPTS}

- Collaborative and social computing; - Human-centered computing; • Robotics;

\section{KEYWORDS}

Personalization, Rehabilitation, Socially Assistive Robots, HumanRobot Interaction, Care Robots

\section{ACM Reference Format:}

Ronit Feingold Polak and Shelly Levy-Tzedek. 2021. Personalized Human Robot Interaction in the Unique Context of Rehabilitation. In Adjunct Proceedings of the 29th ACM Conference on User Modeling, Adaptation and Personalization (UMAP '21 Adjunct), June 21-25, 2021, Utrecht, Netherlands. ACM, New York, NY, USA, 2 pages. https://doi.org/10.1145/3450614.3463415

\section{INTRODUCTION}

The need for effective, scalable rehabilitation strategies is projected to grow substantially in the coming decades, with increased patient survival after diseases with severe functional deficits, such as stroke [1.]. In recent years, many research works studied the applicability of using socially assistive robots (SARs) in different domains such as health, education, and elderly care. Researchers aspire to use SARs to supplement and augment the efforts of educators, parents, caregivers, and clinicians through combined applications of engineering, computer science, and social science [2.]. More recently, and with the progress of artificial intelligence (AI), these mechanisms are being developed to understand and to interact with humans in long-term, real-world settings, exposing many challenges and opportunities [3.]. The field has shown that human-robot partnerships

Permission to make digital or hard copies of all or part of this work for personal or classroom use is granted without fee provided that copies are not made or distributed for profit or commercial advantage and that copies bear this notice and the full citation on the first page. Copyrights for components of this work owned by others than ACM must be honored. Abstracting with credit is permitted. To copy otherwise, or republish, to post on servers or to redistribute to lists, requires prior specific permission and/or a fee. Request permissions from permissions@acm.org.

UMAP '21 Adjunct, fune 21-25, 2021, Utrecht, Netherlands

(c) 2021 Association for Computing Machinery.

ACM ISBN 978-1-4503-8367-7/21/06 . \$ \$15.00

https://doi.org/10.1145/3450614.3463415

\author{
Shelly Levy-Tzedek \\ Ben-Gurion University of the Negev \\ shelly@bgu.ac.il
}

can mitigate critical health challenges that require socially mediated, personalized, long-term support, such as in the contexts of older-persons care and rehabilitation [3.].

\section{PATIENT-CENTERED CARE IN REHABILITATION}

Patient-centered care has been widely accepted as an essential component of healthcare, specifically in rehabilitation [4.]. The importance of tailoring the rehabilitation program for the individual needs of each person was also highlighted by Winstein and Varghese [5.], who noted that researchers and clinicians should adopt a more patient-centered approach in clinical trial design and in clinical care. In rehabilitation, SARs engage with persons from an especially vulnerable population. Patients depend on reliable and effective relationships with their therapists [6.]. They are often older and multimorbid and suffer from psychological distress because of their disability [1.]. Complex injuries-which may entail motor, cognitive, speech, and language impairments- call for a comprehensive interaction design that addresses these aspects [1.].

Rehabilitation, in its nature, is a long and dynamic process. Due to the limitations and frustration a person in the process of rehabilitation (e.g., following a stroke) may experience because of their impairments, a system that is designed for persons with complex conditions should be as personalized as possible.

\section{UNIQUE ASPECTS OF PERSONALIZATION IN HRI FOR REHABILITATION}

We posit that, in addition to the personalization level that can benefit HRI in other contexts, HRI in the context of rehabilitation has to adapt to the user on a much shorter time scale, by adding further layers of adaptation, which, themselves, vary by context. By way of example, we could imagine (on one end of the personalization spectrum) a robotic pet: the user might set a name, a gender and a personality type for it, and never change these personalized settings again. On the other end of the spectrum, we argue, is the user who has had a stroke, and is aided by a SAR in the rehabilitation process: the user encounters the robot first when their functional ability, shortly after the stroke, is impaired. The robot thus needs to suggest exercise sets at an appropriate level, and adjust the level of feedback it provides accordingly. After several weeks, the functional ability of the user might change, and the robot should adapt to their new condition. The user might also fatigue after a short exercise session, to which the robot should be able to respond (e.g., by suggesting a break, or terminating the session). In addition, the user might display an evolving set of compensatory movements in the process of rehabilitation [7.], to which the robot should respond appropriately (e.g., by changing the exercise set). 
Importantly, we argue that the factors that should be personalized would vary, depending on the specific rehabilitative context. E.g., while in post-stroke motor training, one layer of adaptation would be adjusting the training difficulty level to the current motor ability of the user, in cardiac training, the SAR should adapt to their changing levels of physiological signals.

Thus, in order for the robotic device to be effective for therapy and accepted by both the patient and the clinician, it has to be flexible and adjustable, taking into account the complexity of the specific impairment and the dynamic changes that the patient experiences. Following our recent study with expert clinicians [8., 9.] and a long-term study with post-stroke individuals in the wild [9.], we argue that personalization is an essential and crucial component for successful interactions between patients and SARs in the rehabilitation context. The value in adapting the rehabilitation program to the personal needs of the patient was also stressed by the participants in our study, who mentioned the importance of personalizing the design of HRI and Human-Computer Interaction (HCI) and tailoring it to the specific task and patient needs. In our recent study, 10 post-stroke participants exercised with the Pepper robot (Aldebaran, Soft Bank) for 15 sessions over a period of 5-7 weeks [10.]. They noted they would like the system to be able to adapt to their personal performance, e.g. by adapting the feedback to their movement patterns, and by automatically progressing through the exercise-set levels based on their success rates. With the development of AI, when patients encounter a SAR, they expect a highly intelligent system that can automatically adapt to their changing and progressing needs. If these expectations are not met, the interaction may fail.

\section{CONCLUSIONS}

In the context of rehabilitation, personalization is an essential component in establishing trust and in fostering motivation. Furthermore, customizing the feedback a patient receives from the SAR and adapting it to the performance of the individual is an important part of the motor-learning process, and thus of the therapy process. As such, the goal of personalization in rehabilitation is not only to establish engagement, but it is indeed an essential component for the recovery of motor and cognitive abilities over a long-term interaction.

\section{REFERENCES}

[1.] Kellmeyer, P., et al., Social robots in rehabilitation: A question of trust. Science Robotics, 2018. 3: p. eaat1587.

[2.] Matarić, M.J., Socially assistive robotics: Human augmentation versus automation. Science Robotics, 2017. 2(4): p. eaam5410.

[3.] Clabaugh, C. and M. Matarić, Robots for the people, by the people: Personalizing human-machine interaction. Science Robotics, 2018. 3(21): p. eaat7451.

[4.] Bamm, E.L., et al., Exploring client-centered care experiences in in-patient rehabilitation settings. Global Qualitative Nursing Research, 2015. 2: p. 1-11.

[5.] Winstein, C. and R. Varghese, Been there, done that, so what's next for arm and hand rehabilitation in stroke? NeuroRehabilitation, 2018. 43(1): p. 3-18

[6.] Langer, A., et al., Trust in socially assistive robots: Considerations for use in rehabilitation. Neuroscience Biobehavioral Reviews, 2019. 104: p. 231-239.

[7.] Kashi, S., et al., A machine-learning model for automatic detection of movement compensations in stroke patients. IEEE Transactions on Emerging Topics in Computing, 2020.

[8.] Feingold Polak, R., et al., Novel gamified system for post-stroke upper-limb rehabilitation using a social robot: focus groups of expert clinicians, in 2019 International Conference on Virtual Rehabilitation (ICVR). 2019, IEEE. p. 1-7.

[9.] Feingold Polak, R. and S. Levy-Tzedek, Social Robot for Rehabilitation: Expert Clinicians and Post-Stroke Patients' Evaluation Following a Long-Term Intervention, in Proceedings of the 2020 ACM/IEEE International Conference on Human-Robot Interaction. 2020. p. 151-160.

[10.] Feingold Polak, R. and S. Levy-Tzedek, A Robot Goes to Rehab: A Novel Gamified System for Stroke Rehabilitation using a Socially Assistive Robot: Methodology Feasibility Testing. PREPRINT, avilable at Research Square, 2020. 29. 